\title{
Perfil epidemiológico dos pacientes oncológicos com Covid-19 em Alagoas
}

\author{
Epidemiological profile of cancer patients with Covid-19 in Alagoas \\ Perfil epidemiológico de pacientes con cáncer con Covid-19 en Alagoas
}

Dandara Dinna Cavalcante da Silva ORCID: https://orcid.org/0000-0001-8739-0096 Universidade Federal de Alagoas, Brasil Email:dandaracavallcantee@gmail.com

Eugênia Carla Agostinho de Melo ORCID: https://orcid.org/0000-0002-2779-9516 Universidade Federal de Alagoas, Brasil Email:carla.melo.al@gmail.com

Letícia Nawany Tavares de Luna ORCID: https://orcid.org/0000-0002-6609-0163 Universidade Federal de Alagoas, Brasil Email:leticiatavares.ufal@gmail.com

Letícia Marianny Freitas de Oliveira ORCID: https://orcid.org/0000-0003-3861-2953 Universidade Federal de Alagoas, Brasil Email:leticiamarianny8@gmail.com

Gabriel Soares Bádue

ORCID: https://orcid.org/0000-0002-4663-4936 Nome da instituição onde atua, País E-mail: gabriel.badue@ fanut.ufal.br

Alda Graciele Claudio dos Santos Almeida ORCID: https://orcid.org/0000-0003-0406-8849 Universidade Federal de Alagoas, Brasil E-mail: alda.almeida@eenf.ufal.br

\begin{abstract}
Resumo
Objetivo: Analisar o perfil epidemiológico dos pacientes oncológicos acometidos pela Covid-19 em Alagoas. Metodologia: Trata-se de uma pesquisa observacional, descritiva, de abordagem quantitativa, realizada a partir de dados secundários extraídos do "Painel Covid-19 em Alagoas", dos casos confirmados de Covid-19 notificados no período de 08/03/2020 (primeiro caso em Alagoas) a 02/08/2021. Resultados: Neste período, Alagoas confirmou 80 casos de pacientes oncológicos com Covid-19. A predominância de casos confirmados foi no sexo masculino (53\%), 43 pacientes $(53,75 \%)$ apresentaram comorbidades associadas, dentre elas destacam-se hipertensão arterial sistêmica $(46,25 \%)$ e diabetes $(21,25 \%)$. O local de residência destes pacientes foi, predominantemente, a $1^{a}$ macrorregião de saúde, que concentra a capital Maceió. Um dado alarmante foi que todos os pacientes oncológicos evoluíram para óbito em decorrência da infecção por SARS-CoV-2. Conclusão: Conforme a análise dos dados epidemiológicos da Covid-19 em Alagoas, referente aos pacientes oncológicos foi apontado que independente do sexo, da presença ou não de comorbidades e das condições de saúde além do diagnóstico de câncer, essas variáveis não repercutiram nos desfechos clínicos, considerando-se que todos vieram a óbito. Esse resultado leva a crer que a própria condição de imunossupressão dos pacientes oncológicos já é por si só um alto fator de risco para mortalidade quando esses adoecem por Covid-19.
\end{abstract}

Palavras-chave: Covid-19; Oncologia; Perfil de saúde.

\begin{abstract}
Objective: To analyze the epidemiological profile of cancer patients affected by Covid-19 in Alagoas. Methodology: This is an observational, descriptive research with a quantitative approach, carried out from secondary data extracted from the "Covid-19 Panel in Alagoas", from confirmed cases of Covid-19 notified in the period from 03/08/2020 (first case in Alagoas) to 02/08/2021. Results: Alagoas has 80 cancer patients confirmed with Covid-19, of these patients the predominance is male $(53 \%)$, where 43 patients $(53.75 \%)$ have comorbidities, including systemic arterial hypertension $(46,25 \%)$ and diabetes $(21.25 \%)$. The place of residence of these patients was predominantly the $1 \mathrm{st}$ health macro-region, which concentrates the capital Maceió, and of these, all patients died as a result of SARS-CoV-2 infection. Conclusion: According to the analysis of epidemiological data from Covid-19 in Alagoas, regarding cancer patients, it was pointed out that regardless of gender, the presence of comorbidities and health conditions, in addition to the diagnosis of cancer, it did not affect clinical outcomes, considering that all died.
\end{abstract}

Keywords: Covid-19; Medical Oncology; Health profile. 


\begin{abstract}
Resumen
Objetivo: Analizar el perfil epidemiológico de los pacientes oncológicos afectados por Covid-19 en Alagoas. Metodología: Se trata de una investigación de observación, descriptiva con enfoque cuantitativo., realizado a partir de datos secundarios extraídos del "Panel Covid-19 en Alagoas", de casos confirmados de Covid-19 notificados en el período del 08/03/2020 (primer caso en Alagoas) al 02 / 08/2021. Resultados: Alagoas tiene 80 pacientes oncológicos confirmados con Covid-19, de estos pacientes el predominio es masculino (53\%), donde 43 pacientes (53,75\%) presentan comorbilidades, incluyendo hipertensión arterial sistémica (46,25\%) y diabetes (21,25\%). El lugar de residencia de estos pacientes fue predominantemente la $1^{\mathrm{a}}$ macrorregión sanitaria, que concentra la capital Maceió, y de estos, todos los pacientes fallecieron a consecuencia de la infección por SARS-CoV-2. Conclusión: De acuerdo con el análisis de datos epidemiológicos del Covid-19 en Alagoas, respecto a los pacientes con cáncer, se señaló que independientemente del sexo, la presencia de comorbilidades y condiciones de salud, además del diagnóstico de cáncer, no afectaba la clínica. resultados, considerando que todos murieron.
\end{abstract}

Palabras clave: Covid-19; Oncología Médica; Perfil de salud.

\title{
1. Introdução
}

Em dezembro de 2019 foi identificado em Wuhan, capital da província de Hubei, na China, o novo coronavírus, denominado Síndrome Respiratória Aguda Grave Coronavírus-2 (SARS-CoV-2), causador da Covid-19 (Corona Virus Disease 2019) (OMS. 2020). O primeiro caso de Covid-19 em Alagoas data de 08 de março de 2020 (Alagoas, 2021). Desde então, a pandemia por SARS-CoV-2 tornou-se um dos maiores desafios para a sociedade, economicamente e para a saúde (Matuscheck et al., 2020). A Covid-19 é considerada uma emergência de saúde pública, através de pesquisas e coleta de dados observa-se que determinados grupos apresentam um risco maior de desenvolver a doença em sua forma grave. Por meio do levantamento epidemiológico acerca dos indivíduos infectados pelo SARS-CoV-2, identificou-se que a maioria dos casos ocorre em pessoas idosas, com comorbidades ou profissionais da saúde (Souza et al., 2020).

A presença de comorbidades é um fator importante para o desfecho dos pacientes com Covid-19, observa-se que pacientes oncológicos, por apresentarem essa condição, indicam em diversos estudos um alto risco para desenvolver complicações relacionadas à infecção por SARS-CoV-2, devido a imunossupressão decorrente da malignidade da doença ou do tratamento ao qual o indivíduo está sujeito (Liang et al., 2020). Pacientes oncológicos possuem crescimento desordenado e rápido das células tumorais e requerem métodos terapêuticos que tragam controle da doença, redução da metástase e menores sequelas para as pessoas com câncer. Ademais esses pacientes fazem parte do grupo de alto risco para Covid-19 em decorrência da imunossupressão decorrente do tratamento (Ramos, 2020). A terapêutica oncológica através de imunossupressor, com quimioterapia e radioterapia, demanda um acompanhamento estreito, tendo em vista um elevado risco de contágio e agravamento pela Covid-19, dados que constituem um grupo populacional vulnerável à infecção por SARSCoV-2, quando comparados a pacientes não oncológicos (Monteiro et al., 2021). Não obstante, sintomas decorrentes do câncer, como a febre podem mascarar sinais de infecção por Covid- 19 (Matuscheck et al., 2020).

No que tange o isolamento social, esta é uma das ferramentas mais eficazes para contenção da propagação do vírus, e, por conseguinte, sendo os pacientes oncológicos destinados às maiores recomendações de isolamento social (Silva et al., 2020; Aquino et al., 2020). Ademais, dentre umas das manifestações da Covid-19 está a assintomatologia, resultado de uma menor replicação viral; apesar disso, mantém o elevado potencial de transmissão (Zhou et al., 2020).

A limitação do acesso aos serviços de saúde e a ineficácia do tempo para receber os cuidados necessários são outros riscos para pacientes oncológicos. A interrupção ou continuidade do tratamento curativo contra o câncer ainda é um debate controverso, o que denota a necessidade de mais ensaios clínicos antes de qualquer recomendação generalizada. No entanto, recomenda-se que os pacientes que recebem terapia curativa não adiem o tratamento, apesar do risco potencial de infecção por SARS-CoV-2 durante a terapia anticâncer. Interromper o tratamento da doença metastática gera em agravamento do quadro clínico, o que leva o indivíduo a ser admitido apenas para o manejo dos sintomas (Al-Quteimat et al., 2020). 
A inexistência de estudos que tracem o perfil epidemiológico no Estado de Alagoas de pacientes oncológicos que apresentaram a Covid-19 foi um fator determinante na motivação para essa pesquisa. Destarte, apresentar uma descrição do perfil epidemiológico de pacientes oncológicos acometidos pela Covid-19 neste Estado auxiliará nos planejamentos estratégicos direcionados a este público vulnerável. Diante disso, essa pesquisa tem como objetivo descrever o perfil epidemiológico dos pacientes oncológicos acometidos pela Covid-19 em Alagoas.

\section{Metodologia}

Trata-se de uma pesquisa observacional, descritiva, de abordagem quantitativa (Severino, 2018). Realizada a partir de dados secundários extraídos do "Painel Covid-19 em Alagoas", o qual possui acesso público de forma online (Alagoas, 2021). Os dados analisados, referem-se aos casos notificados no período de 08/03/2020, data do registro do primeiro caso de Covid19 em Alagoas, a 02/08/2021. Neste estudo, foram incluídos os casos de Covid-19 notificados em Alagoas, correspondentes às pessoas com diagnóstico de câncer de acordo com as diretrizes do Ministério da Saúde (Ministério da Saúde, 2021).

As variáveis qualitativas analisadas foram: sexo, macrorregião de saúde de residência, comorbidades (doença renal crônica, imunossupressão, pneumopatia, doença neurológica, obesidade, cardiopatia, diabetes e hipertensão arterial sistêmica) e situação do paciente (recuperado, óbito, hospitalizado, isolamento domiciliar).

O presente estudo não foi submetido ao Comitê de Ética em pesquisa, por se tratar de dados secundários, de domínio e acesso público. Todos os dados utilizados são de acessos livre e de utilização pública e não possibilita a identificação do paciente, estando de acordo com a resolução n 466/2012 do Conselho Nacional de Saúde que estabelece normas e diretrizes regulamentadoras de pesquisas envolvendo seres humanos (Ministério da Saúde, 2016).

A extração dos dados foi realizada no segundo dia do mês de agosto do presente ano (Alagoas, 2021). Os dados foram agrupados e analisados no Microsoft Excel@ 2016 - versão 2108.

\section{Resultados}

A partir da análise do banco de dados, foi constatado o registro de 80 pacientes oncológicos diagnosticados com Covid-19 no estado de Alagoas. Os municípios de residência dos pacientes foram distribuídos pelas macrorregiões de saúde do estado, além disso, também foi analisada a presença ou não de comorbidades e a situação do paciente, se recuperado, se hospitalizado, se em isolamento domiciliar ou se óbito. Essas variáveis estão descritas na Tabela 1.

Tabela 1. Distribuição quanto às variáveis: sexo, residência, comorbidade e situação dos pacientes oncológicos diagnosticados com Covid-19 em Alagoas.

\begin{tabular}{lcc}
\hline Variáveis & $\mathrm{n}$ & $\%$ \\
\hline Sexo & 42 & $53 \%$ \\
Masculino & 38 & $47 \%$ \\
Feminino & & \\
Residência & 57 & $72 \%$ \\
$1^{\text {a Macrorregião de Saúde }}$ & 23 & $28 \%$ \\
$2^{\text {a Macrorregião de Saúde }}$ & 23
\end{tabular}




\section{Comorbidade}

$\operatorname{Sim}$

Não
43

37

\section{Situação do paciente}

\begin{tabular}{lcc} 
Recuperado & 0 & $0 \%$ \\
Hospitalizado & 0 & $0 \%$ \\
Isolamento domiciliar & 0 & $0 \%$ \\
Óbito & 80 & $100 \%$ \\
\hline Total de pacientes & 80 & $100 \%$ \\
\hline
\end{tabular}

Fonte: Autores.
$53,75 \%$

$46,25 \%$

A primeira macrorregião de saúde de Alagoas detém o maior percentual dos casos de pacientes oncológicos confirmados de Covid-19 (72\%). Nesta macrorregião de saúde, os municípios que apresentaram notificação de casos foram Atalaia (3), Branquinha (1), Campo Alegre (2), Chã Preta (1), Coruripe (1), Japaratinga (1), Maceió (30), Marechal Deodoro (3), Paulo Jacinto (1), Penedo (2), Pilar (2), Rio Largo (5), Roteiro (1), São Miguel dos Campos (1) e União dos Palmares (3). Na segunda macrorregião de saúde, os municípios com notificações de casos foram: Arapiraca (8), Campo Grande (2), Delmiro Gouveia (1), Girau do Ponciano (2), Joaquim Gomes (1), Major Isidoro (1), Maravilha (1), Mata Grande (1), Palestina (1), Palmeira dos Índios (2), Pão de açúcar (2) e Traipu (1).

No que se refere às comorbidades, 43 pacientes (53,75\%) apresentavam comorbidades associadas ao diagnóstico de câncer, e 37 pacientes (46,25\%) não apresentavam comorbidades. O Gráfico 1 apresenta a distribuição das comorbidades apresentadas pelos pacientes oncológicos diagnosticados com Covid-19 no estado de Alagoas. Analisou-se também a situação dos pacientes e foi observado que os 80 pacientes (100\%) vieram a óbito em decorrência da infecção por SARS-CoV-2.

Gráfico 1. Distribuição quanto às comorbidade dos pacientes oncológicos diagnosticados com Covid-19 em Alagoas.

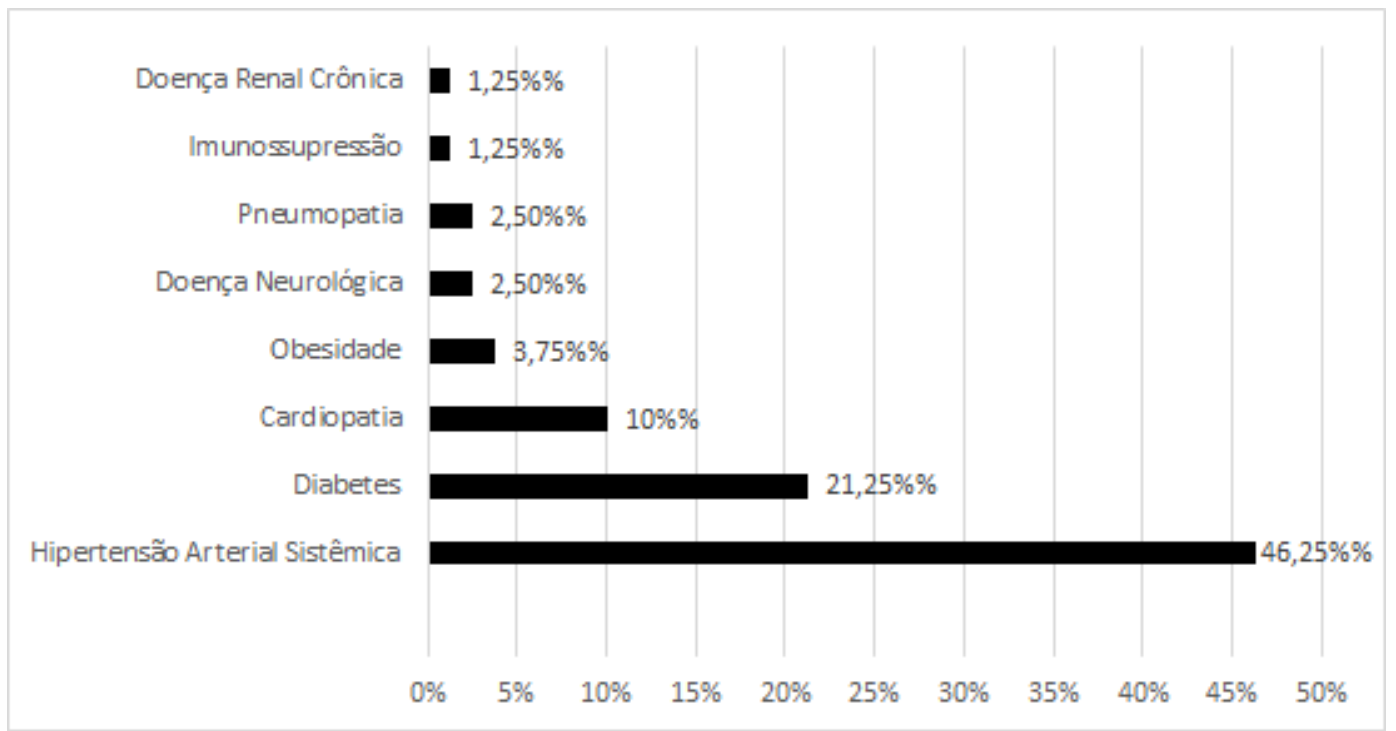

Fonte: Autores. 
Diante do exposto no Gráfico 1, constatou-se que a Hipertensão Arterial Sistêmica (HAS) foi a comorbidade que mais se manifestou, com prevalência de 46,25\% (37 pacientes), seguida pelo diabetes mellitus, representando $21,25 \%$ dos pacientes afetados (17). As cardiopatias estavam presentes em 10\% (8) dos pacientes e a obesidade em 3,75\% (3), as demais estavam presentes em menor número de pacientes oncológicos.

\section{Discussão}

A pandemia da Covid-19 apresenta-se como desafio para prestação de cuidados aos pacientes oncológicos, onde esses já são populações de alto risco e geralmente com comorbidades significativas e imunossupressão sistêmica devido ao tratamento (Viscariello et al., 2020.). Comorbidades como diabetes, HAS e doença cardiovascular, assim como idade avançada, são fatores de risco para Covid-19 (Austrian, 2021).

No tocante às comorbidades, foi constatado que a presença de condições mórbidas simultâneas no diagnóstico de Covid-19 em pacientes oncológicos não influenciou no desfecho clínico. Torna-se evidente, segundo os dados do estudo, que a maior influência é o próprio diagnóstico de câncer. Haja vista que todos os pacientes, independentes de comorbidades, além do câncer, vieram a óbito. Destaca-se que os pacientes oncológicos fazem parte do grupo de alto risco para a Covid-19 diante da imunossupressão secundária ao tratamento ou da imunodepressão relacionada ao adoecimento por câncer (Ramos, 2020).

Pacientes portadores de doença renal crônica (1,25\%) possuem maior risco para infecção grave e desenvolvimento de lesão renal aguda, e consequentemente está associada a desfechos adversos, com maior mortalidade e pior prognóstico. Considerando o alto contágio e risco de infecção, pacientes submetidos à diálise são um grupo vulnerável devido a maior dificuldade em manter o distanciamento e isolamento domiciliar, culminando em maior incidência de complicações e internações hospitalares prolongadas (Pecly et al., 2021).

No que se refere às doenças neurológicas $(2,50 \%)$, as manifestações dos sintomas da infecção podem ser confundidas com manifestações da própria condição do paciente, como a cefaleia. No entanto, também podem aparecer algumas manifestações tardias, como o acidente vascular cerebral, a encefalite, a meningite, a encefalomielite e a mielite aguda (Silva, 2021).

Outro relevante resultado encontrado no presente estudo, refere-se as pessoas com HAS (46,25\%) e diabetes (21,25\%), pesquisas sugerem que estas estão mais suscetíveis à infecção grave ou fatal da Covid-19, por apresentarem níveis elevados da enzima ECA2(Enzima Conversora de Angiotensina 2), a qual o SARS-CoV-2 entra na célula humana. Entretanto, há um conflito, pois, a enzima ECA2 possui um efeito protetor e anti-inflamatório ao epitélio pulmonar (Asturian, 2021).

Além disso, as lesões pulmonares e os danos cardiovasculares (10\%) conforme o gráfico 1, também em sua grande parte são mediados pelo sistema renina-angiotensina. A infecção promove a diminuição dos sítios de ligação da ECA2 para angiotensina II e consequentemente a conversão em angiotensina 1,7, inibindo sua ação anti-inflamatória e vasoconstritora. O agravamento de paciente obesos $(21,25 \%)$ conforme o gráfico 1 , ocorre pela alta secreção de adipocinas e citocinas pró inflamatória pelo tecido adiposo, culminando em desregulação do sistema imune, favorecimento de trombos, metabolismo da glicose ineficiente comprometimento cardiovascular e respiratória (Alonso-Lopes, 2021).

No que se refere ao sexo entre os casos confirmados de Covid-19 em pacientes oncológicos dos registros do Painel Informativo de Covid-19 em Alagoas, o sexo masculino apresentou uma maior frequência. O sexo masculino é o mais acometido pelo SARS-CoV-2 nos registros de hospitalizações e óbitos por Síndrome Respiratória Aguda Grave (SRAG) (BRASIL, 2020). Entretanto, o sexo não se mostrou uma variável atrelada ao desfecho dos pacientes neste estudo. 
Ademais, em um estudo realizado no estado de Sergipe, que visou descrever o perfil sociodemográfico, clínico e principais desfechos dos pacientes adultos e idosos com câncer internados em um hospital universitário (HU), os pacientes oncológicos no contexto da pandemia, $65 \%$ dos pacientes vieram a óbito (Fontes et al., 2021).

Em relação à distribuição dos casos confirmados entre as macrorregiões de saúde, notou-se que $72 \%$ deles se concentram na $1^{\text {a }}$ macrorregião de saúde do estado de Alagoas. Esta região concentra a capital do estado e os municípios da região metropolitana. A capital Maceió é o maior município em população desta macrorregião, com melhores condições socioeconômicas e consequentemente um processo acentuado de urbanização, onde os serviços de saúde não acompanham esse crescimento, especialmente a população mais pobre (Alagoas, 2011).

O presente estudo limita-se aos dados divulgados pela Secretaria de Estado do Planejamento, Gestão e Patrimônio de Alagoas, trazendo apenas sexo, macrorregião de saúde de residência, comorbidades e situação do paciente, o que dificulta a obtenção de informações clínicas e sociodemográficas dos pacientes. Alagoas possui um quantitativo de poucos serviços públicos de assistência à saúde e baixa condição socioeconômica (Teixeira, 2010).

\section{Conclusão}

O presente estudo identificou um dado alarmante no Estado de Alagoas, dos pacientes oncológicos que foram notificados no Painel com Covid-19, todos foram a óbito independente da associação ou não com outras comorbidades, como hipertensão arterial sistêmica, diabetes, cardiopatias, entre outras, visto que dentre os que não apresentavam outras doenças além do câncer também desenvolveram a forma grave da Covid-19 e não resistiram. Esse resultado suscita na suposição de que a própria condição de imunossupressão dos pacientes oncológicos já é por si só um alto fator de risco para mortalidade quando esses adoecem por Covid-19, entretanto estudos minuciosos devem ser realizados para confirmação dessa suposição assim como a investigação de outras causas relacionadas a mortalidade desse público-alvo quando infectados pelo Sars-Cov-2.

Dadas as limitações do presente estudo, sobretudo em relação as limitações e deficiências nas notificações de comorbidades dos pacientes, a equipe deste artigo recomenda trabalhos futuros, que ao adotarem metodologia semelhante realizem comparações com achados na literatura, revelando as especificidades dos perfis epidemiológicos traçados.

\section{Referências}

Alagoas. (2011). Plano Diretor de Regionalização Da Saúde do Estado de Alagoas - PDR/AL - Revisão no Ano 2011. Secretaria de Estado da Saúde. Superintendência de Planejamento e Participação Social. Disponível em: https://www.saude.al.gov.br/wp-content/uploads/2020/06/pdr_2011.pdf

Alagoas. (2021) Secretaria de Estado da Saúde de Alagoas (SESAU). Painel Covid-19 em Alagoas. Disponível em: http://bit.ly/Covid19AL

Alonso-Lopes, B, Furieri, LB, \& Alonso-Vale, M. I. C. (2021). Obesity and covid-19: a reflection on the relationship between pandemics. Revista Gaúcha de Enfermagem, 42(spe). https://doi.org/10.1590/1983-1447.2021.20200216

Al-Quteimat, OM, \& Amer, AM (2020). O impacto da pandemia de COVID-19 em pacientes com câncer. American Journal of Clinical Oncology , 43 (6), 452-455. https://doi.org/10.1097/COC.0000000000000712

Aquino, E. M., Silveira, I. H., Pescarini, J. M., Aquino, R., Souza-Filho, J. A. D., Rocha, A. D. S., \& Lima, R. T. D. R. S. (2020). Medidas de distanciamento social no controle da pandemia de COVID-19:potenciais impactos e desafios no Brasil. Ciência \& SaúdeColetiva,25(suppl 1), 2423-2446

Asturian, K. (2021). O papel dos inibidores da enzima conversora de angiotensina e dos antagonistas dos receptores de angiotensina em pacientes com Covid19: uma revisão narrativa. Rev. Ciênc. Méd., (Campinas), 215035-215035. https://pesquisa.bvsalud.org/portal/resource/pt/biblio-1150810

Comentários do Diretor-Geral da OMS no briefing para a mídia em 2019-nCoV. Organização Mundial da Saúde. 11 de fevereiro de 2020. Disponível em: https://www.who.int/dg/speeches/detail/who-diretor-geral-s-remarks-at-the-media-briefing-on-2019-ncov-on-11-february-2020

Fontes Júnior, J.., Silva, Érika R.., Almeida, LA da H.., Santos, CN dos, Souza, JLS de., Bezerra, DJ., Matos, CJO, \& Tofani, PS (2021). Perfil sociodemográfico e clínico de pacientes com câncer no contexto da pandemia de COVID-19 em um Hospital Universitário do Nordeste: estudo transversal. Pesquisa, Sociedade e Desenvolvimento , 10 (11), e255101119611. https://doi.org/10.33448/rsd-v10i11.19611 
Research, Society and Development, v. 11, n. 1, e22511124691, 2022 (CC BY 4.0) | ISSN 2525-3409 | DOI: http://dx.doi.org/10.33448/rsd-v11i1.24691

Liang, W, Guan, W. \& Chen, R. Cancer patients in SARS-CoV-2 infection: a nationwide analysis in China. Lancet Oncol. 2020;21(3):335-7. doi: https://doi.org/10.1016/S1470-2045(20)30096-6

Matuscheck, C., Fischer, J. C. \& Combs, S. E. (2020). Medidas de prevenção de infecção e incidência de infecções por SARS-CoV-2 em pacientes com câncer submetidos à radioterapia na Alemanha, Áustria e Suíça. Strahlenther Onkol. 196, 1068-79 . https://doi.org/10.1007/s00066-020-01681-1

Melo, A.C., Thuler, L. C. S., \& Silva, J. L. (2020). Cancer in patients with COVID-19: A report from the Brazilian National Cancer Institute. PLoS One. Journal pone. doi:10.1371/journal.pone.024126

Ministério da Saúde. (2021). Guia de Vigilância Epidemiológica Covid-19. https://www.gov.br/saude/pt-br/coronavirus/publicacoes-tecnicas/guias-eplanos/guia-de-vigilancia-epidemiologica-covid-19/view

Monteiro, D.E., Fialho, I. C. T. S., Passos, P.M. \& Fuly, P.S.C. (2021). Gestão do enfrentamento dos riscos da COVID-19 em uma rede ambulatorial oncohematológica: relato de experiência. Rev. Bras. Enferm. 2021;74: e20201080. https://doi.org/10.1590/0034-7167-2020-1080

Nabuco, G., Pires de Oliveira, M. H. P., \& Afonso, M. P. D. (2020). O impacto da pandemia pela COVID-19 na saúde mental: qual é o papel da Atenção Primária à Saúde?. Revista Brasileira De Medicina De Família E Comunidade, 15(42), 2532. https://doi.org/10.5712/rbmfc15(42)2532

Pecly, I. M. D., Azevedo, R. B., Muxfeldt, E. S., Botelho, B. G., Albuquerque, G. G., Diniz, P. H. P., Silva, R. \& Rodrigues, C. I. S. (2021). COVID-19 e doença renal crônica: uma revisão abrangente. Jornal Brasileiro de Nefrologia, 43, 383-99. https://doi.org/10.1590/2175-8239-JBN-2020-0203

Ramos, RS (2020). A Enfermagem Oncológica no Enfrentamento da Pandemia de Covid-19: Reflexões e Recomendações para a Prática de Cuidado em Oncologia. Revista Brasileira De Cancerologia, 66., e-1007. https://doi.org/10.32635/2176-9745.RBC.2020v66nTemaAtual.1007

Resolução $n^{\circ}$. 510, de 07 de abril de 2016. Dispõe sobre as normas aplicáveis a pesquisas em Ciências Humanas e Sociais cujos procedimentos metodológicos envolvem a utilização de dados diretamente obtidos com os participantes ou de informações identificáveis ou que possam acarretar riscos maiores do que os existentes na vida cotidiana, na forma definida nesta Resolução. Recuperado de https://www.in.gov.br/materia//asset_publisher/Kujrw0TZC2Mb/content/id/22917581

Severino, A. J. (2018). Metodologia do trabalho científico. São Paulo: Cortez.

Silva, L. L. S. D., Lima, A. F. R., Polli, D. A., Razia, P. F. S., Pavão, L. F. A., Cavalcanti, M. A. F. D. H., \& Toscano, C. M. (2020). Medidas de distanciamento social para o enfrentamento da COVID-19 no Brasil: caracterização e análise epidemiológica por estado. Cadernos de Saúde Pública, 36, e00185020.

Silva, F. S. C. A. da, Bucur, A., Rosado, S. N., Balhana, S. dos S., \& Meneses-Oliveira, C. M. (2021). Neurological dysfunction associated with COVID-19. Revista Brasileira de Terapia Intensiva, 33(2). https://doi.org/10.5935/0103-507x.20210042

Souza, J. B., Conceição, V. M., Araújo, J. S., Bitencourt, J. V. O. V., Silva, F. C. C. \& Rossetto, M. (2020). Câncer em tempos de COVID-19: repercussões na vida de mulheres em tratamento oncológico. Revista Enfermagem, 28, e51821. DOI: http://dx.doi.org/10.12957/reuerj.2020.51821

Teixeira, C. F. (1999). Epidemiologia e planejamento de saúde. Ciência \& Saúde Coletiva, 4(2), 287-303. https://doi.org/10.1590/s1413-81231999000200005

Viscariello, N., Evans, S., Parker, S., Schofield, D., Miller, B., Gardner, S., Fong de Los Santos, L., Hallemeier, C., Jordan, L., Kim, E., \& Ford, E. (2020). A multi-institutional assessment of COVID-19-related risk in radiation oncology. Radiotherapy and oncology: journal of the European Society for Therapeutic Radiology and Oncology, 153, 296-302. https://doi.org/10.1016/j.radonc.2020.10.013

Zhou, R., Li, F., Chen, F., Liu, H., Zheng, J., Lei, C., \& Wu, X. (2020). Viral dynamics in asymptomatic patients with COVID-19.International Journal of Infectious Diseases, 96, 288-290. 